\title{
Depression beim lokalisierten Prostatakarzinom
}

Hintergrund und Fragestellung: Eine Androgenentzugstherapie (ADT) kann zu einer Depression beitragen. Eine ursächliche Beziehung lies sich bislang jedoch nicht feststellen. Retrospektiv wurde daher untersucht, ob die Aufnahme einer ADT und/oder deren längere Dauer beim Prostatakarzinom (PCa) mit einem erhöhten Risiko einer Depression einhergeht.

Patienten und Methodik: Gestützt auf die SEER-Datenbank wurden 78.552 über 65-jährige Männer identifiziert, die in der Zeit zwischen 1992 und 2006 an einem PCa der Stadien I-III erkrankt waren. Patienten mit psychiatrischen Diagnosen im vorausgegangenen Jahr wurden ausgeschlossen. Die primäre Analyse galt dem Zusammenhang zwischen einer pharmakologischen ADT und der Diagnose einer Depression bzw. der Durchführung einer ambulanten oder stationären psychiatrischen Behandlung. Daten zur Wahl der medikamentösen Behandlung der Depression standen nicht zur Verfügung. In der sekundären Analyse wurde die Assoziation zwischen der Dauer einer ADT und dem Auf-

\section{Originalie}

Dinh KT et al. Association of Androgen Deprivation Therapy With Depression in Localized Prostate Cancer. J Clin Oncol. 2016;34(16):1905-12. treten einer Depression betrachtet.

Ergebnisse: Insgesamt entwickelten $43 \%$ der Patienten (n = 33.882) unter einer ADT im Vergleich zu Patienten ohne ADT eine höhere, kumulative
3-Jahres-Inzidenz einer Depression (7,1 vs. 5,2\%) mit stationärer (2,8 vs. $1,9 \%)$ oder ambulanter (3,4 vs. 2,5\%) psychiatrischer Behandlung; alle Berechnungen hochsignifikant ( $<<0,001)$. Adjustierte Cox-Analysen zeigten, dass Patienten mit im Vergleich zu ohne ADT ein um $23 \%$ erhöhtes Risiko hatten, eine Depression zu bekommen (adjustierte Hazard Ratio [aHR] 1,23, $95 \%$-Konfidenzintervall [95\%-KI] 1,15-1,31), ein 29\% höheres Risiko für eine stationäre (aHR 1,29, $95 \%$-KI 1,17-1,41) und eine nicht signifikante Risikoerhöhung um $7 \%$ für eine ambulante psychiatrische Behandlung (aHR, 1,07, $95 \%$-KI 0,97-1,17). Das Risiko einer Depression stieg mit der Dauer der ADT von $12 \%$ bei $\leq 6$ Monaten Behandlung, $26 \%$ bei $7-11$ Monaten auf $37 \%$ bei $\geq 12$ Monaten $\left(\mathrm{p}_{\text {Trend }}\right.$ von $<0,001$ ). Entsprechende Effekte wurden bei stationären $\left(\mathrm{p}_{\text {Trend }}<0,001\right)$ und ambulanten $\left(\mathrm{p}_{\text {Trend }}<0,001\right)$ Patienten unter psychiatrischer Behandlung beobachtet.

Schlussfolgerungen der Autoren: Die Ergebnisse dieser großen Studie an älteren Männern mit lokalisiertem PCa zeigen, dass eine pharmakologische ADT das Risiko steigert, eine Depression zu entwickeln. Dieses Risiko nimmt mit der Dauer der ADT kontinuierlich zu. Es wird daher gefordert, die möglichen psychiatrischen Nebenwirkungen der ADT in die Aufklärung der Patienten mit einzubeziehen, bevor eine solche Therapie begonnen wird, und sie kontinuierlich im Blickfeld zu behalten.

Schlüsselwörter: Prostatakarzinom - Androgenentzugstherapie - ADT - Depression - unerwünschte Arzneimittelwirkungen

\section{- Kommentar von Ulrich R Kleeberg, Hamburg \\ „Gerade für Ältere ist die ADT eher belastend"}

Die Zahl der mit einer Androgenentzugsherapie (ADT) adjuvant wie palliativ behandelten Prostatakarzinompatienten hat mit den Erfolgen der operativen und strahlentherapeutischen Interventionen und der hierdurch signifikant verbesserten Lebenserwartung kontinuierlich zugenommen und erreicht inzwischen an die 50\% [1]. Diese sich über Jahre und regelhaft bis zum Tode hinziehende Therapie ist mit einer Reihe von $\mathrm{Ne}$ benwirkungen verbunden, die sich mit zunehmenden Alter der Betroffenen zu einer relevanten geriatrischen Multimorbidität mit deutlicher Einschränkung der Lebensqualität addieren. Sie umfasst metabolische, kardiovaskuläre, sexuelle, ossäre und gelenksbezogene sowie kognitive Belastungen. Hinzu kommen psychomotorische und psychiatrische, speziell depressive Folgen. In ihrer Gesamtheit wächst damit die Gefahr, dass die PCa-Patienten nicht an dem Tumor, sondern an der Dekompensation der Begleitmorbidität versterben. Die große Bedeutung, die tägliche Bewegung für die Stabilisierung des Tumorleidens und dessen Besserung hat, wird durch die wachsende Komorbidität zusätzlich eingeschränkt.

In diesem Licht ist die wichtige Empfehlung, eine ADT erst noch sorgsamer Beobachtung der Tumorbiologie und des ger- iatrischen Gesamtstatus zurückhaltend aufzunehmen, von vitaler Bedeutung. Sie bedarf insbesondere bei den Betagten einer obligaten geriatrisch-internistischen Begleitung. Initial eine aufmerksame Beobachtung oder aktive Überwachung des PCA zu versuchen sowie unter einer palliativen ADT therapiefreie Intervalle einzufügen, mindert die erwähnten Risiken und Einschränkung der Lebensqualität.
Literatur
1. Gilbert SM et al. Urol Oncol. 2011;29(6):647-53.

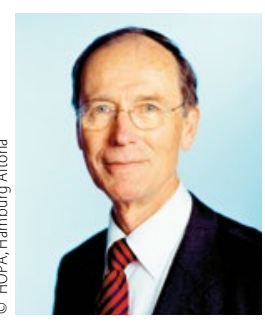

Prof. Dr. Ulrich R. Kleeberg

Hämatologisch-onkologische

Praxis Altona, Tagesklinik Struensee-Haus,

Hamburg

urkleeberg@hopa-hamburg.de 